\title{
Non-neoplastic enterogenous submucosal cystic lesion of the colon
}

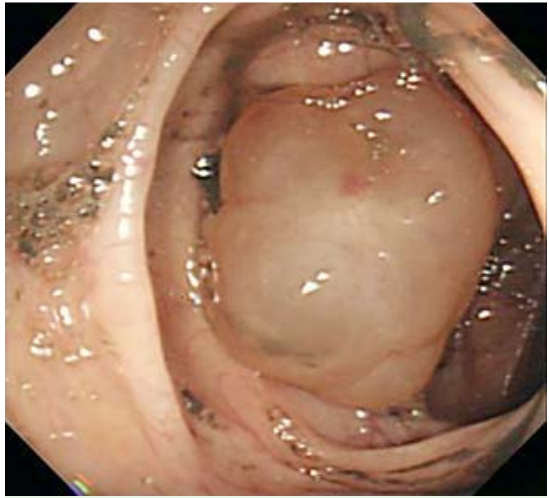

Fig. 1 A polypoid lesion located $60 \mathrm{~cm}$ from the anal verge in the descending colon, near the splenic flexure.

Non-neoplastic enterogenous submucosal cystic lesions arise from the colonic or rectal wall, and more common ones include duplication cysts, lymphangiomas, and colitis cystic profunda [1]. An enterogenous cyst may detach itself from the intestinal wall and lie close to its point of origin, which may be in the intermuscular, submucosal, or subserosal layers of the intestinal wall. Accurate diagnosis of nonneoplastic enterogenous submucosal cystic lesions is difficult, and thus the preoperative diagnosis mainly depends on initial colonoscopy findings, which may provide information that supports the abnormal clinical findings [2].

We report a case of non-neoplastic colonic submucosal cystic lesion, discovered incidentally in a 62-year-old man. The patient had undergone sigmoidoscopy, which revealed abnormal findings related to a submucosal lesion in the descending colon. The surface was smooth and resembled that of a cyst ( $\bullet$ Fig. 1); a non-neoplastic colonic enterogenous submucosal cystic lesion was suspected. At exploratory laparotomy, the lesion was removed along with segmental resection of the descending colon. Gross examination of the resected specimen showed a grayish, elastic, monolocular cystic lesion measuring $4 \times 3.5 \times 0.8 \mathrm{~cm}$, with a well-defined and smooth wall ( Fig. 2). Histological examination revealed a multiloculated, submucosal cystic lesion lined by atrophic epithelium and lymphocytic inflamma-

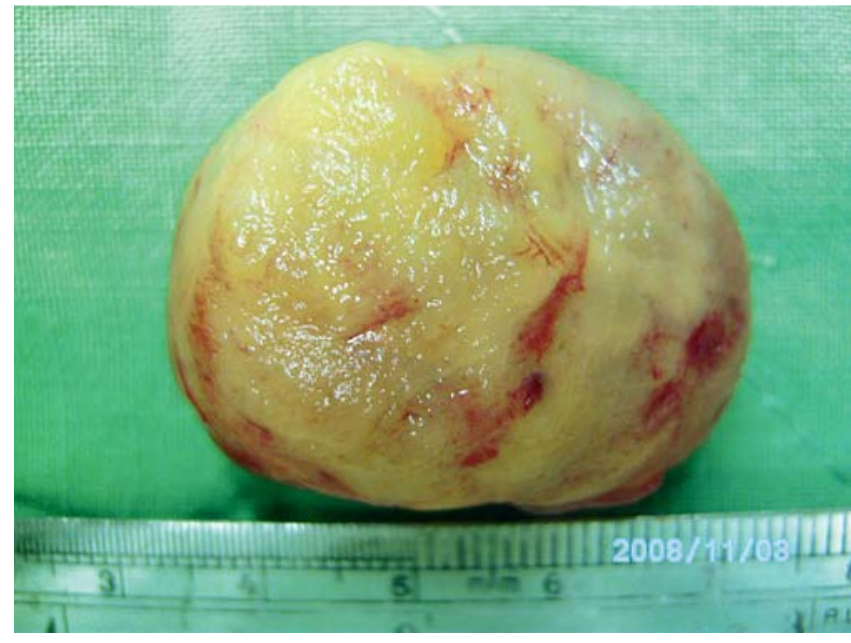

Fig. 2 The grayish, elastic, monolocular cystic lesion measured $4 \times 3.5 \times 0.8 \mathrm{~cm}$. The walls were smooth and well defined.

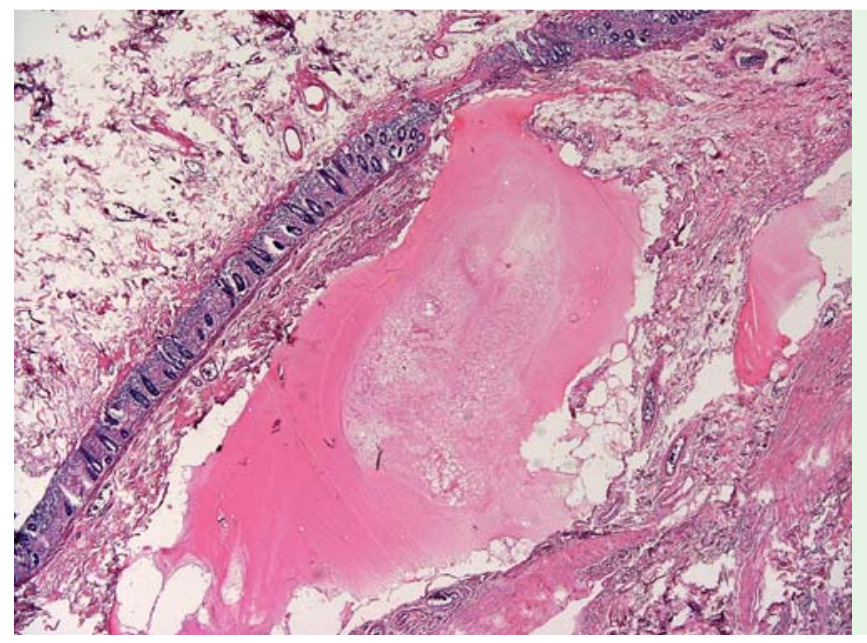

Fig. 3 Multiloculated submucosal cystic lesions contain serous fluid (hematoxylin and eosin, magnification $\times 20$ ).

tory infiltrate in the edematous stroma ( Fig. 3). The histopathologic diagnosis was colonic cyst.

Non-neoplastic enterogenous submucosal cystic lesions are uncommon and present in a variety of ways. If a lesion is suspected to be a non-neoplastic enterogenous submucosal cyst, it should be completely excised for local control of symptoms and to prevent the risk of obstruction, septic sequelae, and malignant change $[3,4]$. However, with regard to nonspecific symptoms and the clinical presentation of such lesions, there is a need for further prospective and retrospective studies to elucidate the prognosis and ideal treatment of these lesions.

Endoscopy_UCTN_Code_CCL_1AD_2AJ

\section{Y. S. Yeh ${ }^{1,2}$, W. T. Huang ${ }^{3}$, C. H. Kuo ${ }^{4,5}$, J. Y. Wang ${ }^{1,6,7,8}$}

1 Division of Gastroenterology and General Surgery, Department of Surgery, Kaohsiung Medical University Hospital, Kaohsiung Medical University, Kaohsiung, Taiwan

2 Department of Emergency Medicine, Department of Trauma, Kaohsiung Medical University Hospital, Kaohsiung Medical University, Kaohsiung, Taiwan Department of Pathology, Kaohsiung Medical University Hospital, Kaohsiung Medical University, Kaohsiung, Taiwan

4 Department of Internal Medicine, Kaohsiung Medical University Hospital, Kaohsiung Medical University, Kaohsiung, Taiwan 
${ }^{5}$ Department of Internal Medicine, Faculty of Medicine, College of Medicine, Kaohsiung Medical University, Kaohsiung, Taiwan

6 Department of Surgery, Faculty of Medicine, College of Medicine, Kaohsiung Medical University, Kaohsiung, Taiwan

7 Graduate Institute of Medicine, College of Medicine, Kaohsiung Medical University, Kaohsiung, Taiwan

8 Graduate Institute of Medical Genetics, College of Medicine, Kaohsiung Medical University, Kaohsiung, Taiwan

\section{References}

1 Pickhardt PJ, Kim DH, Heise CP et al. Evaluation of submucosal mucosal lesions of the large intestine. Part 2. Nonneoplastic causes. Radiographics 2007; 27: 1693-1703

2 Rajah S, Ramanujam TM, Tin M et al. Duplication of the rectum: report of four cases and review of the literature. Pediatr Surg Int 1998; 13: $373-376$

3 Pickhardt PJ, Kim DH, Heise CP et al. Evaluation of submucosal mucosal lesions of the large intestine. Part 1. Neoplasm. Radiographics 2007; 27: 1681-1692

4 Sato K, Maekawa T, Sugiyama $\mathrm{N}$ et al. Cystic lymphangiomas of the colon. J Gastroenterol 1999; 34: 520-524

\section{Bibliography}

Dol $10.1055 / \mathrm{s}-0029-1215316$

Endoscopy 2009; 41: E317-E319

(c) Georg Thieme Verlag KG Stuttgart · New York . ISSN 0013-726X

\section{Corresponding author}

\section{J. Y. Wang, MD, PhD}

Department of Surgery

Faculty of Medicine

College of Medicine and Division of Gastroenterology and General Surgery Department of Surgery

Kaohsiung Medical University Hospital Kaohsiung Medical University

100 Tzyou 1st Road

San-Ming District

Kaohsiung 807

Taiwan

Fax: +886-7-3114679

cy614112@ms14.hinet.net;

cy614112@gmail.com 